\title{
Failure Analysis and Design of Cu-Be Alloy Clips
}

\author{
Frederick E. Schmidt, Jr. * \\ * Engineering Systems Inc., 3851 Exchange Avenue, Aurora, IL 60504
}

An investigation of clip failures attributed mainly to premature corrosion fatigue, has been made on $\mathrm{Cu}-1.92 \%$ Be alloy (CDA-172) case clips. The failures were determined to be caused by three factors: (a) excessive localized tensile stresses in the fork area between spring tines, Figure A; (b) microstructure and over-aging of the associated heat treatment, RHC 38-42, Figures D, E, and F for various aging conditions; and (c) crack initiation locations (side and fork regions), Figure B, and fractography, Figure C. Improvement of each of these three factors eliminated the very short service life. Design modifications were made that resulted in a significant cost reduction, and illustrates the value added of a complete design review in addition to simply conducting a root cause failure analysis.

Design Base Case -The life expectancy initially was 2 to 8 weeks of service at line speeds up to 75 feet per minute and temperatures from RT to $300^{\circ} \mathrm{F}$. At least $30 \%$ of the clips were broken by process abuse related to misalignment of the carrier chain on the transfer case. Typical clip life ranged from a low of 63,500 to a high of 96,000 cycles. The majority of the clip failures were caused by the spring leg branches of the conveyor clips progressively cracking due in part to the exposure to a sulfuric acid rinse and dry process cleaning

Quantitative Hardness, HRC Values - Hardness measurements for aging at $1.5 \mathrm{hrs}$. @ 750 ${ }^{\circ} \mathrm{F}$ indicated that both new and over-aged clips ranged from HRC from 37.3 to 38.8 average, while improved clips ranged from HRC from 39.9 to 40.5 after $2.5 \mathrm{hrs}$. @ 625 $\mathrm{F}$. The associated microstructures are shown in Figures D, E, and F. Clearly, the lower temperature aging condition was beneficial in that massive precipitates were no longer forming at the grain boundaries to the extent that corrosion assisted fatigue was dominant.

Modified Clip Design - After correcting the aging treatment to $625^{\circ} \mathrm{F}$ for 2 to 2.5 hours, clip life doubled from 200 to 250,000 cycles. A stress analysis calculation indicated that concentrated tensile stresses were being focused at the center of the fork, and the twisting action of case release promoted side wall fatigue crack initiation. It was decided to reduce the cross-sectional area of the tuning fork clips by $25 \%$ and $50 \%$ by removing the middle of the tine. This had the advantage to reduce the resistance to twisting as the component casings were extracted from the conveyor clips. The actual shape of the tines was changed from straight to hour glass shaped. The hour glass shape change reduced the fork stresses and stress levels in the tines to less than $\sim 40,000$ psi.

Conclusion - The combined affect of eliminating the over-aged structure and reducing the radius and tine stress concentrations resulted in attainment of the endurance limit of $10^{8}$ cycles. Together these changes did not increase the cost per clip@\$1.20 each, but resulted in reduced maintenance and downtime. The net savings per year per production line was $\$ 130,000$ per shift. During peak production years the cumulative savings were $\$ 1.2$ million per year. A discussion of Guinier Preston Zones will be made to explain the mechanism of proper metallurgical aging treatments. 


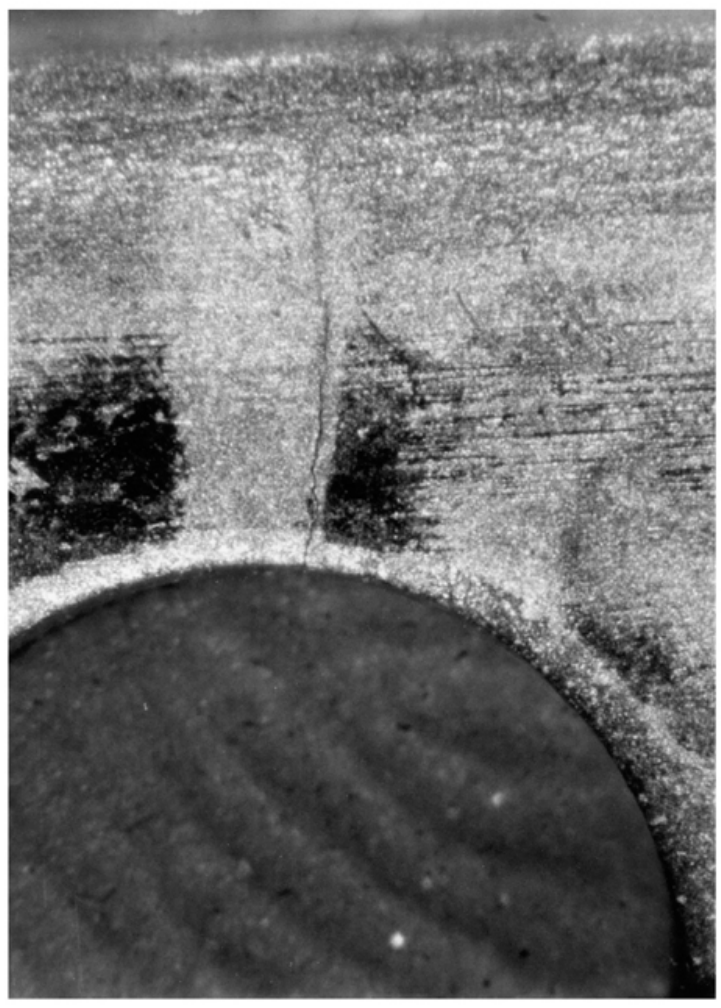

A - Initial Crack, 20x

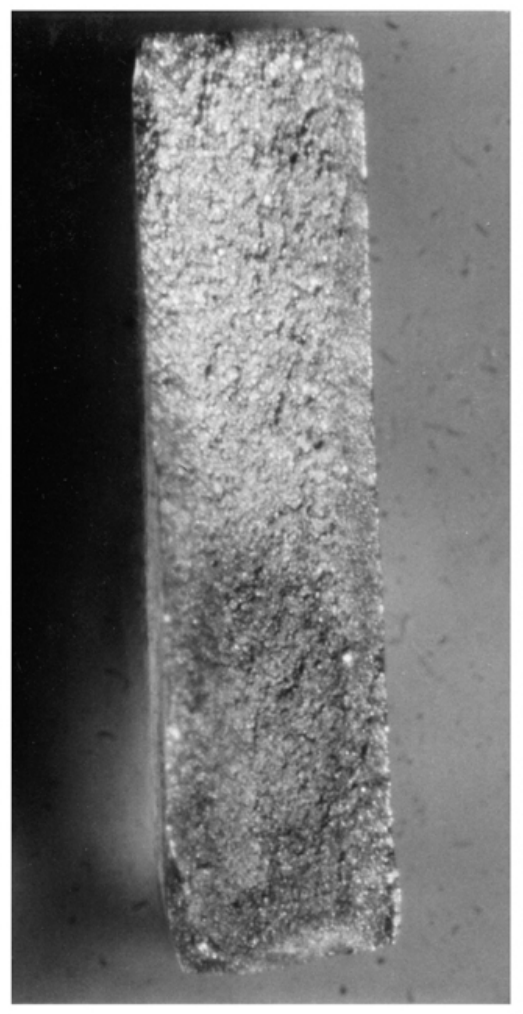

B - Fracture, 40x

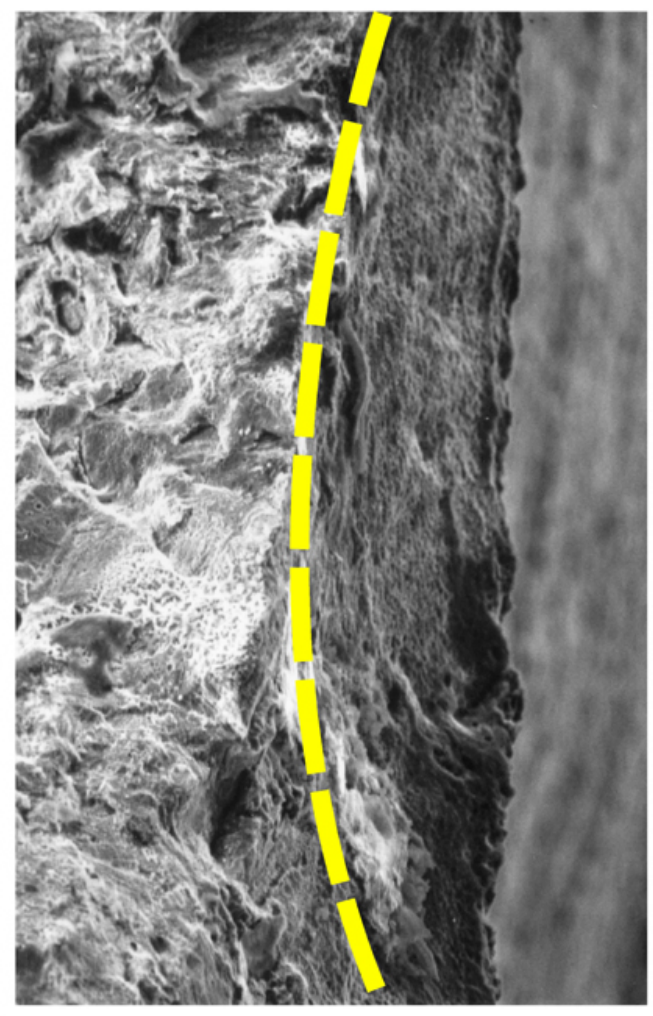

C - SEM - Fatigue, $372 x$

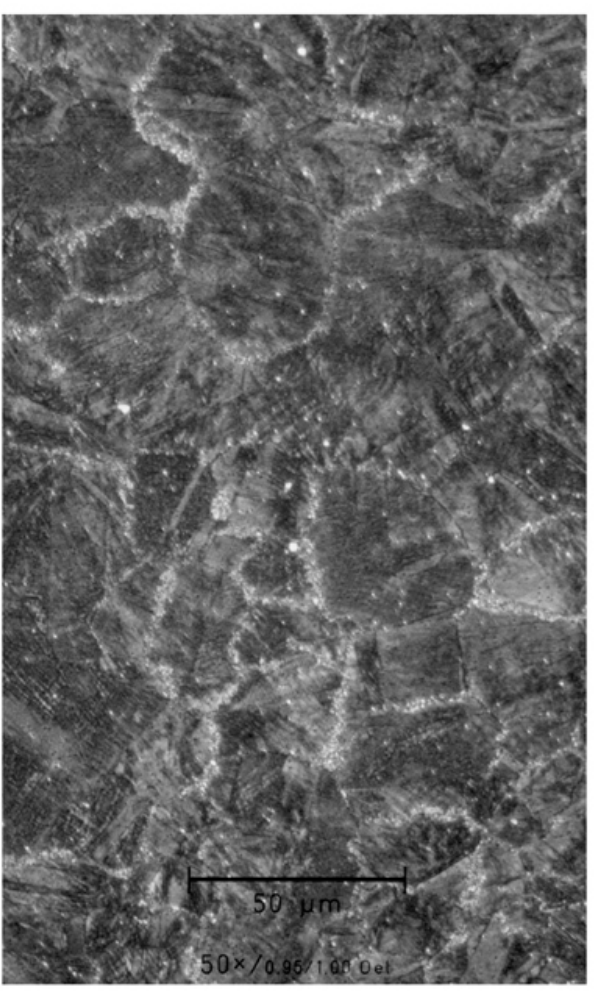

D - Overaged, 500x

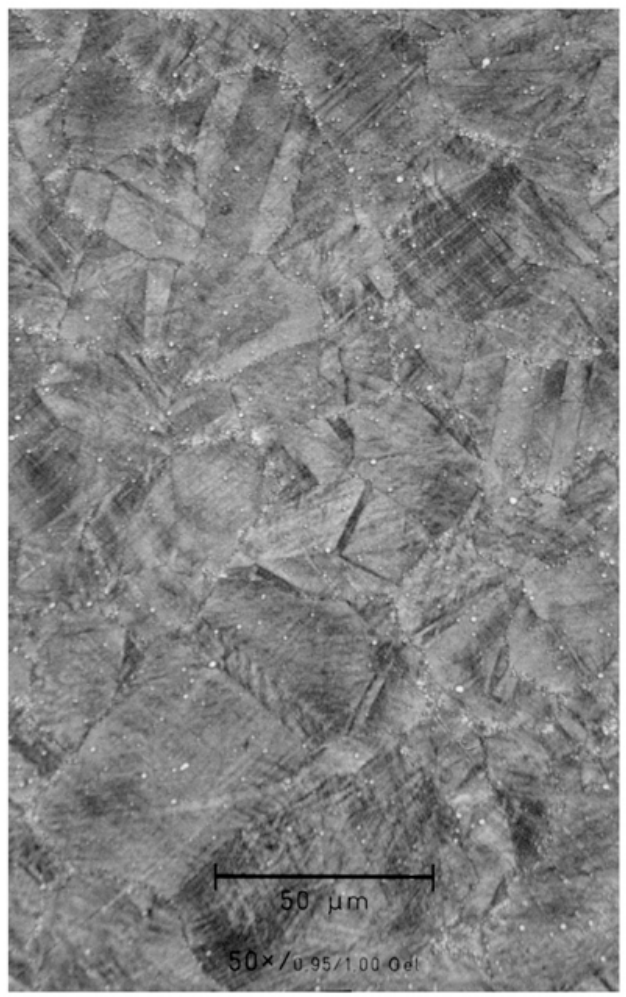

E - 1.5 hrs @ 700 F, 500x

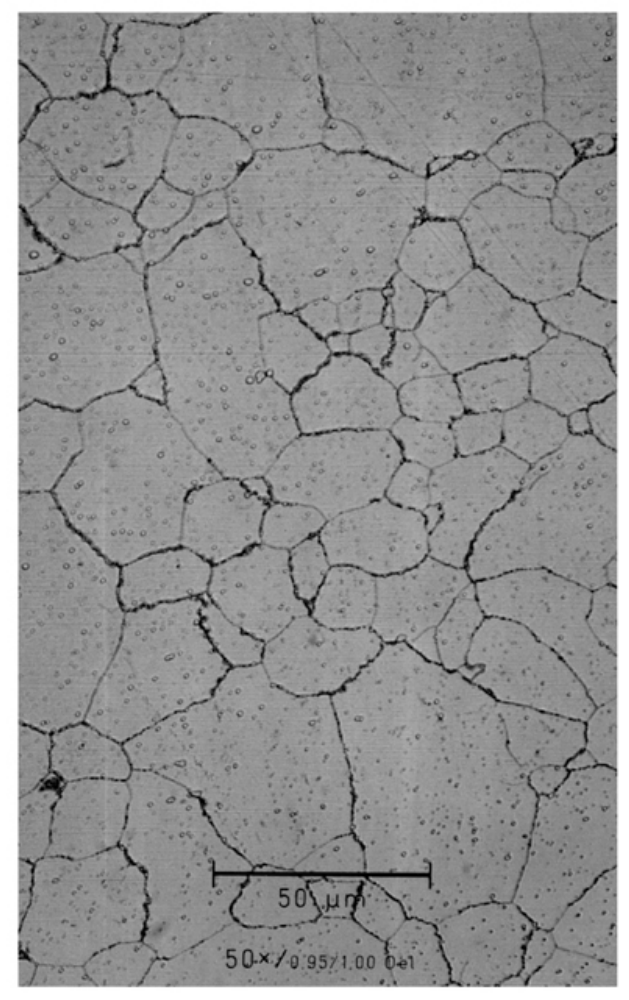

E - 2.5 hrs @ 625 F, 500x 